Plant Tissue Cult. \& Biotech. 27(1): 63-76, 2017 (June)

$\overline{\text { PTC\&B }}$

\title{
Characterization of Progenies from Intergeneric Hybridization Between Oryza sativa L. and Porteresia coarctata (Roxb.) Tateoka
}

\author{
Tasmia Islam, Sudip Biswas, Umme Habiba Mita, R.H. Sarker', \\ M. Sazzadur Rahman', M. Ansar Ali², K.M.S. Aziz ${ }^{3}$ and \\ Zeba I. Seraj*
}

Department of Biochemistry and Molecular Biology, University of Dhaka, Dhaka-1000, Bangladesh

Key words: Porteresia coarctata, Induced-tetraploid, Intergeneric hybridization, SSR marker

\begin{abstract}
Porteresia coarctata (Roxb.) Tateoka is an endemic halophyte growing all over the coastal belt of Bangladesh, propagating through rhizomes and setting a few ricelike grains. So exploiting the genetic potential of this wild rice as salt tolerant donor in possible wide crosses with rice $(2 n=24)$ could be useful. We attempted intergeneric hybridization between Oryza sativa L. and P. coarctata. The survival rate of hybrid progenies in embryo culture was low but among them 2 hybrid plants were successfully matured from the intergeneric cross between the cultivated induced tetraploid of rice, Latisail $(2 \mathrm{n}=4 \mathrm{x}=48)$ and $P$. coarctata $(2 \mathrm{n}=$ 48). The hybrid plants could be successfully established in soil and were not like either of the parents in morphology although some of their features were similar to their maternal parent, Latisail $(4 \mathrm{x})$. Both of the hybrids were investigated through physiological analysis under salinity stress and molecular analyses with rice specific SSR markers. Molecular analysis of the $F_{1}$ DNA with only 3 SSR markers, RM581, RM20224 and RM25271, out of 36 others tested, showed bands specific to both of the parents, while all had common bands with the maternal parent. Dendrogram analysis of the hybrids with the 36 SSR markers, show that $P$. coarctata forms a different clade and is clearly separated from Latisail and the hybrids. The putative hybrids however made a subgroup with Latisail. These observations could be possibly explained if chromosome loss of the paternal
\end{abstract}

*Author for correspondence: <zebai@du.ac.bd>. ${ }^{1}$ Department of Botany, University of Dhaka, Dhaka-1000, Bangladesh. 'Bangladesh Rice Research Institute, Gazipur, Bangladesh. 'International University of Business Agriculture and Technology, Uttara, Dhaka-1230, Bangladesh. 
parent had occurred or may be it was a pleotropic effect of intergeneric hybridization. Physiological screening of the hybrid progenies at the $F_{2}$ generation in seedling stage showed better result in leaf damage score (LDS) and salinity tolerance than their maternal parent Latisail (4x) at $150 \mathrm{mM}$ salt stress for 10 days. $F_{2}$ plants from one of the hybrid plants $(\mathrm{H}-2)$ showed better performance but there was a large variation in response from each of the individual progenies. So, it is likely that some of the salt tolerant characteristics of the pollen parent might have been transferred to the recipient Latisail $(4 \mathrm{x})$. For introgression of better salt tolerant loci from $P$. coarctata, more wide hybrids will need to be produced and repeatedly crossed with $P$. coarctata.

\section{Introduction}

Porteresia coarctata (Roxb. Tateoka is a wild halophytic rice that grows in coastal and tidal regions of Bangladesh, India, Pakistan and Myanmar (Menguer et al. 2017). In Bangladesh, this spontaneously grows from south eastern part, Teknaf to the south western part, Satkhira. Thus the entire coastal region of Bangladesh is home to this halophytic rice. Porteresia locally called "Uri dhan", grows happily and completes its life cycle in an average $20-40 \mathrm{dS} / \mathrm{m}$ of salinity. It is a tetraploid $(2 n=48)$ and sets grains on a single unbranched panicle only once a year around October or November (Latha et al. 2004). The seeds get readily dehydrated, dehisce pre-maturely, do not germinate and are non-viable. So it propagates naturally through rhizomes. The species can also withstand submergence with saline water for quite a long period like 10 - 11 hrs everyday (Garg et al. 2013).

Because of its unique ecological habitats in the saline estuaries and its associated salt tolerance, great curiosity has been aroused in understanding the mechanism of its salt tolerance. Studies conducted on Porteresia coarctata shows it to have very efficient salt glands in the leaves (Flowers et al. 1990). It, therefore can maintain its $\mathrm{K}^{+} / \mathrm{Na}^{+}$ratio for the proper maintenance of its cellular metabolism (Sengupta et al. 2009). At the same time, it possesses many important metabolic enzymes (which have homologues in rice) which are resistant to salt denaturation. It also has an efficient photosynthetic machinery as well as detoxification mechanism which also protects it from salt malfunction (Sengupta et al. 2010). Genomic level investigation has just led to its transcriptome being published under control and sea water exposure (Garg et al. 2013). So, it offers a rich source of germplasm, which gives rise to the possibility of introducing some of its salt tolerance traits into salt sensitive cultivated rice.

Wide or distant hybridization, or breeding between individuals of different species or genera, provides a way to combine diverged genomes into one nucleus. The use of wide (interspecific and intergeneric) hybridization as a 
method for crop improvement is long-established and widely practiced. Advances in intergeneric crosses in tomato (Rick et al. 1986), species of wild grasses from the genera Aegilops, Agropyron and Secale (Fedak 1985), wheat; rapeseed (Brassica napus) and tobacco (Nicotiana tabacum) (Goodman et al. 1987); lymegrass (Cox et al. 2002), sunflower (Helianthus annuus L.) (VassilevskaIvanova et al. 2014) have been well documented. In cereal breeding program there is a very popular method called the Hordeum bulbosum (L.) method (Devaux 2003). By the bulbosum method during wide hybridization some of the genes/portion of genes from one species are transferred to another species and retained by chromatin introgression but due to genetic incompatibility uniparental chromosome might be lost (Gernand et al. 2005).

As $P$. coarctata is good sources of gene(s) for salt tolerance, we had made several attempts to transfer the salt-tolerant character to cultivated rice, through wide hybridization method as a part of the pre-breeding exploration for rice improvement. Successful pollen growth was observed after hybridization when $P$. coarctata was used as the female parent in wide crosses with O. sativa (Sarker et al. 1993). The progenies from these crosses retained the look and properties of $P$. coarctata but showed amplification of both genome specific regions in some chromosomes. And further investigation of those hybrids revealed loss of the O. sativa chromosomes in 2 - 3 generations (Seraj et al. 1996). A few seed were set when induced tetraploid of some $O$. sativa cultivars were used to cross with $P$. coarctata, but did not survive later (Seraj et al. 1996).

Attempts at hybridization of $O$. sativa with $P$. coarctata resulted in production of sterile progenies (Jelodar et al. 1999). Recently however, the IRRI website has reported one fertile progeny out of 34,000 crosses between IR56 and P. coarctata. This news has inspired us to refocus in this research program. So, we had started to collect $P$. coarctata from different locations of Bangladesh in order to attempt its wide hybridization with rice.

This paper reports on 2 successful hybrid plants produced by wide hybridization between $P$. coarctata and $O$. sativa induced tetraploid (Latisail). Their distinct phenotype and genetic similarity to both parents indicated that these plants may have had some genome introgression from $P$. coarctata indicating the rotentiality of this approach. Hybrid plants were completely characterized for their salt tolerant features in response to salt stress. Moreover, generation advancement of one of the hybrid plants showing distinctly different morphology for each progeny showing pleiotropic effects or recombination before chromosomal loss of the alien genotype. 


\section{Materials and Methods}

The materials used in hybridization were comprised of rice cultivar Latisail $(2 \mathrm{n}=4 \mathrm{x}=48)$ and wild rice, Porteresia coarctata $(2 \mathrm{n}=48)$. P. coarcatata was collected from Cox's Bazar district, Bangladesh. Two accession of $P$. coarctata one from Bakkhali and another from Teknaf used in this research were propagated in normal soil in pots for 1 - 2 years in the net house of the Plant Biotechnology Lab, University of Dhaka. Latisail $(2 n=2 x=24)$, a Bangladeshi rice variety was germinated in tissue culture and meristematic zones treated with cotton soaked in colchicine (Blakeslee et al. 1937). New shoots which were much thicker and larger in size were separated and placed in auxin-containing media with colchicine for root growth. Rooted plants were acclimated in hydroponics and then grown in soil. Seeds were collected over several generations (Seraj ZI unpublished data).

Latisail $(4 \mathrm{x})$ and $P$. coarctata was allowed to grow until panicle maturity. Immediately prior to flowering, the panicles of the donor $P$. coarctata were cut and soaked in water for $3 \mathrm{hrs}$ (between 11 a.m. and 2 p.m.) and emasculation of the recipient Latisail $(4 \mathrm{x})$ was accomplished at the same time. Emasculated panicles were covered with oil paper to prevent external pollination. Pollen were collected in a Petri plate by smoothly sliding one panicle with another. The pollen were then dusted on to the stigma of the recipient plant by a small brush and the panicles were then covered again. Immature seeds were harvested in day 10 to 12 and mature seeds were harvested in day 21 from crosses after pollinations. Gibberellic acid (75 ppm) was sprayed every $24 \mathrm{hrs}$ after crossing for 6 consecutive days. This was done particularly to avoid spikelet shattering in the maternal rice parent, Latisail $(4 \mathrm{x})$. After harvesting, seeds were sterilized and germinated in semi-solid MS (1/4th strength) medium. The hybrids germinated in more than 14 days and took more than $4-6$ weeks to grow to about $10-12 \mathrm{~cm}$ Fig. 1C. Thereafter, the seedlings were transferred to hydroponics (Yoshida et al. 1976) for 3 - 4 weeks and then transplanted to soil Fig. 1D. Mature hybrid plants were confirmed for the presence of $P$. coarctata genome by rice specific SSR marker-based PCR.

Genomic DNA was isolated from (0.5 - $1.0 \mathrm{~g})$ pooled leaf tissue of hybrids at $\mathrm{F}_{1}$ generation with parents Latisail $(4 \mathrm{x})$ and $P$. coarctata using the modified CTAB method (Doyle 1987) and was quantified using the Nanodrop spectrophotometer (Nanodrop 1000). The quality of the DNA was assured by checking in $0.8 \%$ agarose gel in TAE buffer. A total of 36 pairs of SSR primers were used to amplify DNA from the leaves. The distribution of the selected SSR primers was even throughout the 12 rice chromosomes. The 36 markers were selected on the basis of the genome-wide distribution from a previous list of highly polymorphic 
83 markers identified in earlier studies on different rice landraces (Seraj ZI, unpublished data). PCR analysis was carried out in a $15 \mu$ l reaction mixture containing $100 \mathrm{ng}$ of plant DNA, $100 \mu \mathrm{M}$ of each dNTP, $2.4 \mathrm{ng}$ of forward and reverse primers each, 1.0 unit of Taq DNA polymerase (Invitrogen, Carlsbad, CA, USA), $1.6 \mathrm{mM} \mathrm{MgCl} 2$, DMSO 2.6\% and $1 \times$ PCR buffer- $\mathrm{MgCl}_{2}$ (Invitrogen). The mixture was then denatured at $95^{\circ} \mathrm{C}$ for 5 mins, followed by 35 cycles of denaturation at $95^{\circ} \mathrm{C}$ for $1 \mathrm{~min}, 1 \mathrm{~min}$ of annealing at $55-60^{\circ} \mathrm{C}$ (depending on primer's Tm), $1 \mathrm{~min}$ of extension at $72^{\circ} \mathrm{C}$ with a final extension step at $72^{\circ} \mathrm{C}$ for $7 \mathrm{~min}$. The $36 \mathrm{RM}$ markers were obtained commercially from idt-1st base, Singapore. The amplified PCR products were resolved and visualized in nondenaturing $10 \%$ polyacrylamide gel electro-phoresis. The gels were stained in $\mathrm{EtBr}$ and visualized with alpha imager gel documentation system. For the microsatellite DNA fingerprinting of the hybrids along with parents Latisail $(4 \mathrm{x})$ and $P$. coarctata, polymorphisms were scored according to their molecular weight on polyacrylamide gels by the Alpha Ease FC imaging system (www.alphaimager.com). The genotyping was done using the Powermarker Software (Liu et al. 2005). The selected dataset is genotype data, the "unknown" is gametic phase for the data type, and the missing numeric is $-9 /-9$. Cluster analysis was based on similarity matrices using the Unweight Pair Group Method with arithmetic mean (UPGMA) (Sneath et al. 1973) and the relationship between cultivars was visualized as a dendrogram using Powermarker and MEGA5. The UPGMA tree was constructed by using the frequency-based distance for the "shared allele" (Chakraborty et al. 1993).

The phenotypic screening for the salinity tolerance at seedling stage was done by the method described by (Amin et al. 2012). Screening was done on $F_{2}$ hybrid plants. In the screening, female parent Latisail $(4 \mathrm{x})$, tolerant control Pokkali and sensitive control IR29 were also used. The seedlings were allowed to grow in Yoshida (Yoshida et al. 1976) culture solution until they reached the four-leaf stage (14 - 18 days after germination). $\mathrm{NaCl}$ stress was applied gradually starting from 5 to $15 \mathrm{dS} / \mathrm{m}$ at 24 hourly increments of $2 \mathrm{dS} / \mathrm{m}$. An increase of leaf number and length was measured after an interval of 4 days up to 16 days or until $90 \%$ of the leaves of the sensitive control were damaged. The tolerance related traits (Leaf damage score or LDS, chlorophyll content and electrolyte leakage) of all stressed plants were then measured. The level of salinity tolerance was evaluated mainly based on the value of LDS, which is based on the percentage of the leaf damage. The plants were scored according to the protocol mentioned by (Amin et al. 2012). The chlorophyll content and electrolyte leakage of the stressed and control hybrids shoots as well as Latisail (4x) were measured at this stage (Yasmin et al. 2016). 
All statistical analyses were done using Data Analysis ToolPak of Microsoft Office Excel 2007 and R package. The F test was performed to verify equal variance of the independent set of samples and the test and ANOVA was performed based on the result assuming equal variance or unequal variance as applicable to compare significant differences $(p>0.05)$ between the stressed and control hybrid plants along with Latisail $(4 \mathrm{x})$.

\section{Results and Discussion}

A total of 1191 spikelets were pollinated in the wide crosses. The results of seed set and number of survived hybrids produced from the crosses are presented in Table 1. Sarker et al. (1993) found that $P$. coarctata accepts $O$. sativa $(2 \mathrm{n}=2 \mathrm{x})$ pollen readily but not vice versa. But in this study, hybrid seeds were produced when Latisail (4x-c) accepted pollen from $P$. coarctata (Fig. 1A). Only two hybrid seeds from wide crosses successfully developed into mature and vigorous plants named $\mathrm{H}-1$ and H-2. The height of the developing hybrid plants was intermediate compared to both the parents (Fig. 2). This suggests that characters from both parents were initially expressed. Apart from their heights, their seed morphology was unlike either parent but more like the seed appearance of Latisail (2x). The $F_{1}$ hybrid plants were extremely slow growing which is a characteristic of $P$. coarctata.

Introgression of some of the genes of $P$. coarctata into Latisail $(4 \mathrm{x}-\mathrm{c})$ rice were confirmed by specific SSR marker-based PCR studies. Comparison of the banding patterns of parents and putative hybrids revealed that some of the Rice Microsatellkite (RM) markers that were polymorphic showed bands specific to both parents (Fig. 3A). For instance, putative hybrids showed both the parental types of banding patterns for RM581, RM20224 and RM25271 out of 36 markers that were tested. Putative hybrids were advanced to the $F_{2}$ generation for observing the effects of salt stress on the seedling stage. For analysis of genetic relatedness between putative hybrids and both parents, a dendrogram was constructed (Fig. 3B) based on their SSR alleles. From the dendrogram (Fig. 3B) it was clearly shown that putative hybrids, Latisail (2x), Latisail $(4 x)$ is in one clade and $P$. coarctata in a different clade. The putative hybrids $(\mathrm{H}-1$ and $\mathrm{H}-2)$ made a subgroup with Latisail.

Seedling stage screening of the putative hybrids at $F_{2}$ generation were performed with one of the parent Latisail $(4 x)$ and Latisail $(2 x)$. To find if any of the genome introgression of $P$. coarctata into the hybrid plants included halotolerant genes, salinity stress $(\mathrm{NaCl})$ of $15 \mathrm{dS} / \mathrm{m}$ was applied to both hybrids (H-1 and H-2) along with their corresponding parent plants (Latisail). IR29 and Pokkali were also included as sensitive and tolerant controls, respectively in the 
seedling stage screening. Hybrid plants (H-1 and $\mathrm{H}-2)$ showed better phenotypic characteristics compared to the control Latisail (4x), Latisail (2x) and IR29 under salt stress (Fig. 4A). H-2 seedlings were, however, observed to be more vigorous and healthy comparedto $\mathrm{H}-1$. In addition, the chlorophyll content was significantly higher in hybrid plants compared to their parent Latisail (4x) (Fig. 4B).
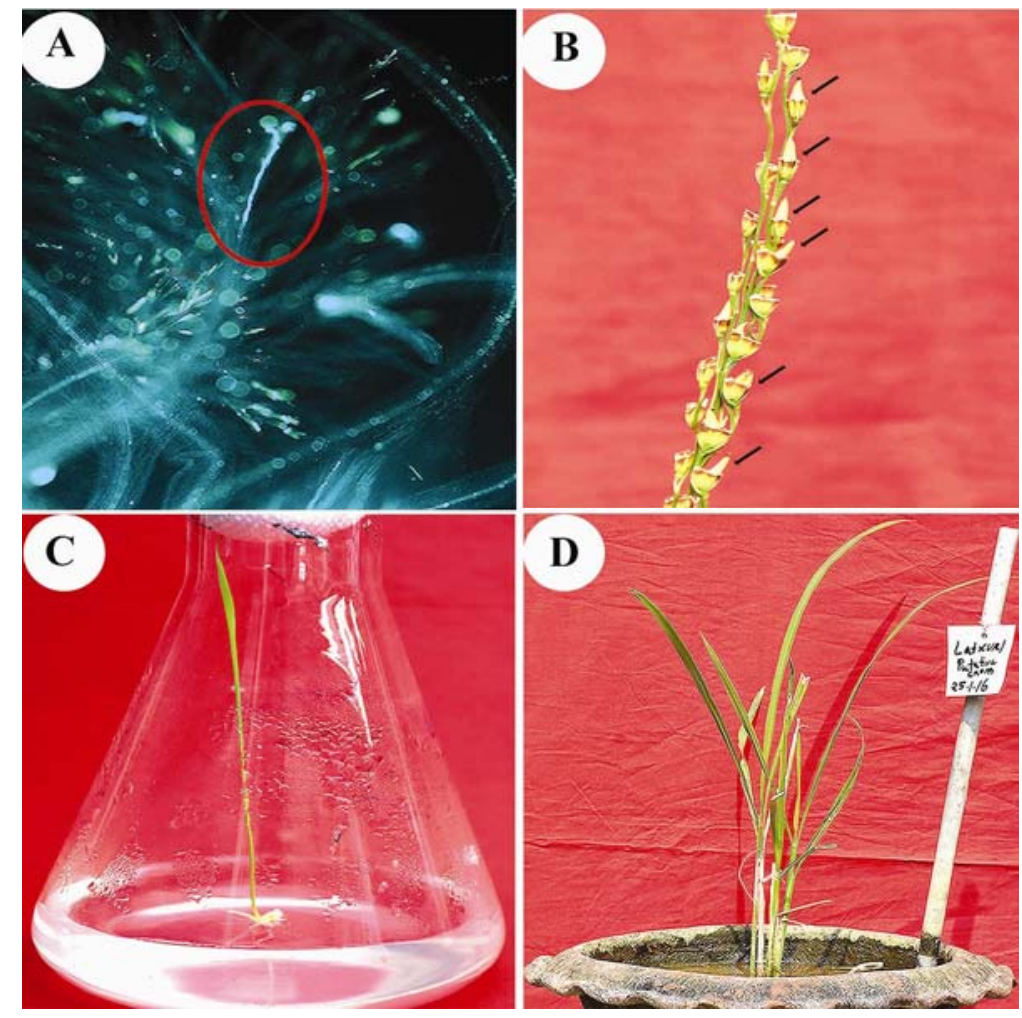

Fig. 1. Wide hybridization between $P$. coarctata and Latisail $(4 \mathrm{x})$. A. Pollen tube growth within the style visualized through fluorescence microscope. B. Developing seeds (arrows) following hybridization. C. Germination and growth of mature embryo in $1 / 4$ strength MS till root and shoot formation. D. Seedlings transferred to soil.

Both hybrids, H-1 and H-2, showed significantly lower leaf damage score (LDS) compared to their one of the parent Latisail $(4 \mathrm{x})$ in salt stress. In hybrid, H-2 showed lower LDS compared to H-1, which was statistically significant (Fig. $5 \mathrm{~A})$.

Lower per cent of electrolyte leakage was recorded in all plants with no significant difference between hybrids and Latisail plants under non-stress conditions (Fig. 5B). The percentage of $\mathrm{H}-1$ and $\mathrm{H}-2$ hybrid plant was significantly lower compared to Latisail plants $(p<0.05)$ after $15 \mathrm{dS} / \mathrm{m}$ salinity treatment for 10 days (Fig. 5B). However, H-1 hybrid plants showed significantly 
higher electrolyte leakage with respect to percentage electrolyte leakage of $\mathrm{H}-2$ hybrid plants.
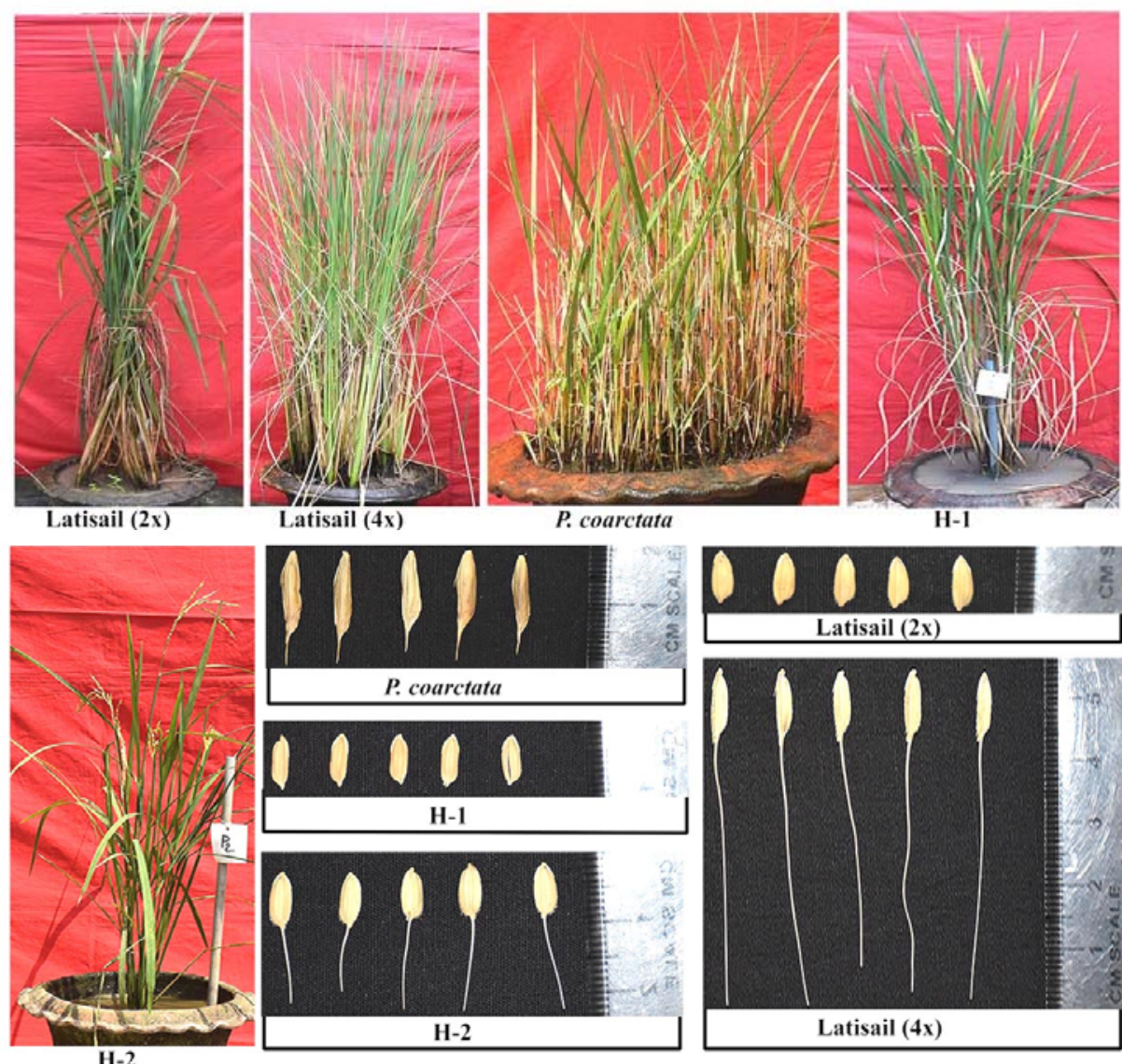

Fig. 2. Formation of hybrids after wide hybridization. $P$. coarctata is short in stature. Its seeds were comparatively taller in size $(\sim 16 \mathrm{~mm})$, un-healthy and needle-like at the tip but without awns; Latisail (4x) was taller in stature. Its seeds were taller $(\sim 11 \mathrm{~mm})$ than Latisail $(2 \mathrm{x})$, healthy with presence of extremely long awns. Latisail $(2 x)$ plants are even taller than Latisail $(4 x)$. Its seeds were smaller $(\sim 8 \mathrm{~mm})$ than Latisail $(4 \mathrm{x})$, healthy and without awn. Putative hybrids were intermediate in height among their parents. H-2 seeds were comparatively short in size $(\sim 7 \mathrm{~mm})$, healthy and with short awns compared to those of $P$. coarctata, but the size of $\mathrm{H}-1$ hybrid seeds were similar to the size of Latisail (2x), un-healthy and without awn.

Survival test of 2-week-old $\mathrm{F}_{2}$ seedlings of hybrid plant $(\mathrm{H}-2)$ showed that they remained green and healthy in hydroponic solution even after 20 days of treatment (150 $\mathrm{mM} \mathrm{NaCl}$ stress). But parent plants Latisail (4x) turned yellow, then brown (Fig. 6A).

Survived $\mathrm{H}-2$ hybrid plants after $150 \mathrm{mM} \mathrm{NaCl}$ stress which were transferred to soil showed distinct phenotypic characteristics from Latisail $(4 \mathrm{x})$. Each $\mathrm{F}_{2}$ 
hybrid plant was different in height (Fig. 6B), with difference in flowering time, variances in seed color, shape and dissimilarity in awn size (Fig. 6C). The awn length of any $\mathrm{F}_{2}$ hybrids did not match with the awn length of Latisail (4x). These results can be explained as pleiotropic effects.

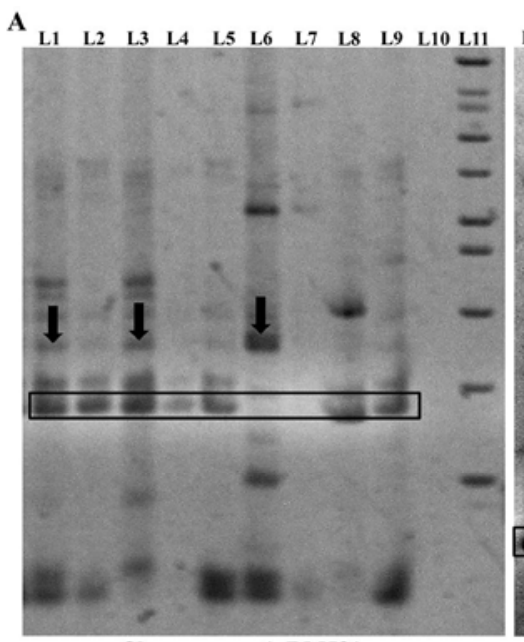

Chromosome-1, RM581

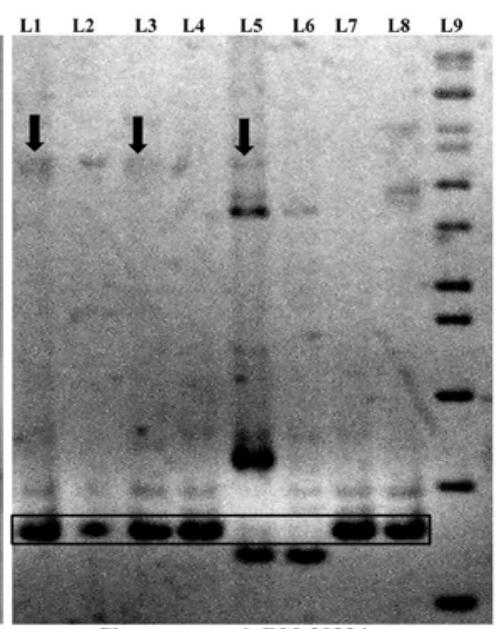

Chromosome-6, RM-20224

B

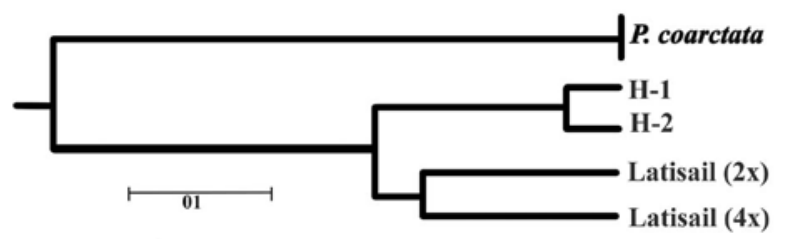

Fig. 3. Banding pattern of RM markers for putative hybrids, parents ( $P$. coarctata and Latisail $(4 x))$ and Latisail (2x) from chromosome 1 and 6. A. Banding pattern for RM marker 581 from chromosome-1. L11: ladder, L1: H-1 and L3: H-2, L6: P. coarctata, L8: Latisail (2x) and L9: Latisail (4x). Here, both of the hybrids showed bands common to both parents. Banding pattern for RM marker 20224 from chromosome-6. L9: ladder, L1 and L3: H-1 and H-2 respectively, L5: P. coarctata, L7: Latisail (2x) and L8: Latisail (4x). Here, again both the hybrids showed bands common to both parents. B. Dendrogram constructed based on the SSR polymorphism of $P$. coarctata (Bakkhali and Teknaf), Latisail $(4 \mathrm{x})$, Latisail $(2 \mathrm{x})$ and putative hybrids using the 36 markers used in the study. In the dendrogram hybrids, Latisail (2x), Latisail (4x) were in one clade and $P$. coarctata in different clade.

In sunflower and lymegrass wide hybridization has successfully been used to produce new cultivars with useful traits of both parents and to incorporate desirable traits of one species into another (Cox et al. 2002, Vassilevska-Ivanova et al. 2014). In disparity, the use of wild gene transfer via wide crosses has not been as successful so far. At the same time, advances in intergeneric gene transfer 


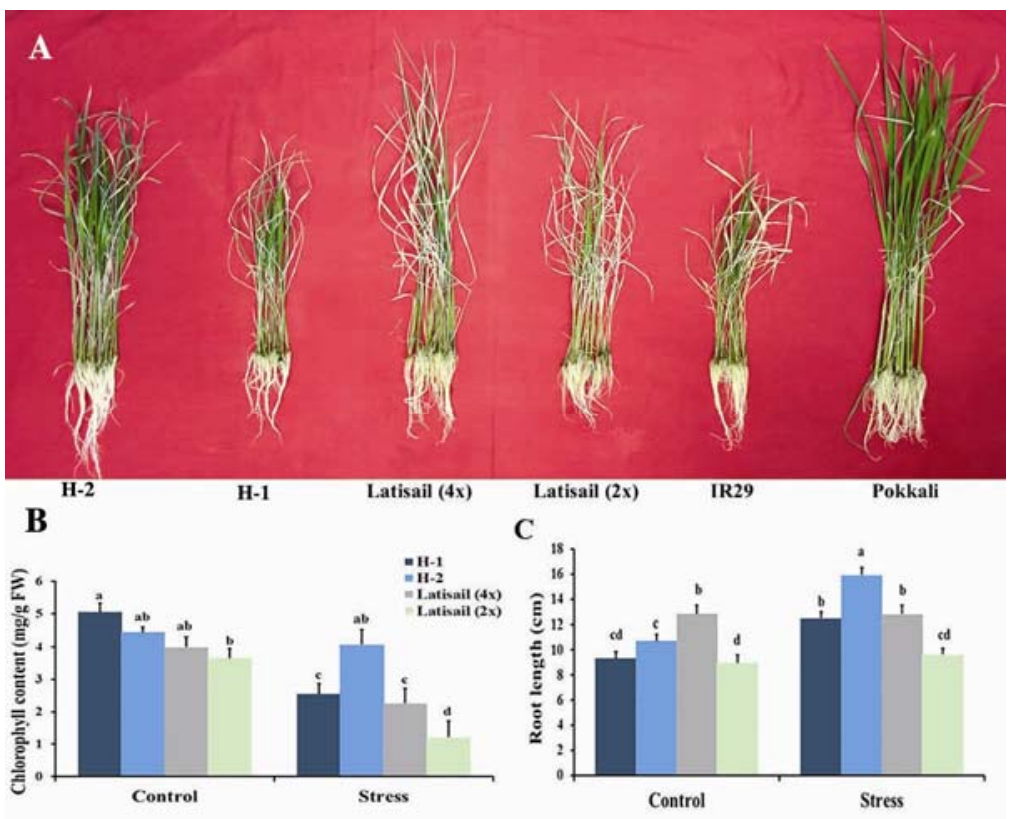

Fig. 4. Seedling stage screening of hybrid plants at $\mathrm{F}_{2}$ generation. A. Phenology of the hybrids and Latisail plants after $15 \mathrm{dS} / \mathrm{m} \mathrm{NaCl}$ stress in hydroponics (From left to right: H-2, H-1, Latisail (4x), Latisail (2x), IR29 and Pokkali). The growth of the hybrid H-2 was better compared to hybrid H-1 under salt stress. B. Chlorophyll content in Latisail plants and hybrids seedlings after $\mathrm{NaCl}$ stress at $15 \mathrm{dS} / \mathrm{m}$ in hydroponics. C. Root length of hybrid and Latisail plants after $15 \mathrm{dS} / \mathrm{m}$ $\mathrm{NaCl}$ stress in hydroponics. Hybrid plants' roots were much longer than the parent Latisail plants. Each bar represents the mean \pm SE $(n=6)$. Different letters in each graph $(a-d)$ indicate significant differences $(\mathrm{p}<0.05$, ANOVA and DMRT).

A

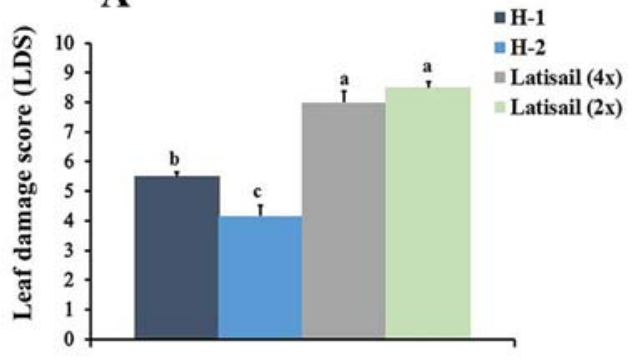

B

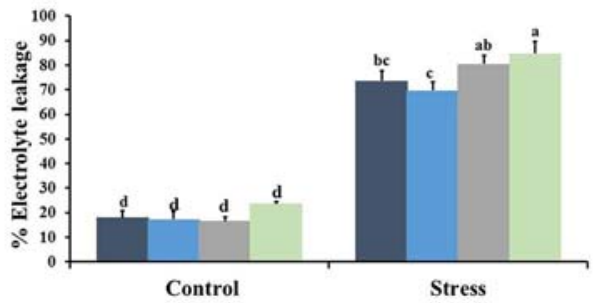

Fig. 5. Leaf damage score and electrolyte leakage in hybrids compared to Latisail plants. A. LDS in hybrids and Latisail seedlings under after $\mathrm{NaCl}$ stress at $15 \mathrm{dS} / \mathrm{m}$ in hydroponics. Each bar represents the mean \pm SE $(n=12)$. LDS was significantly lower in both the hybrid plants. B. Percentage of electrolyte leakage (EL) in hybrids and Latisail seedlings in normal and after $\mathrm{NaCl}$ stress at $15 \mathrm{dS} / \mathrm{m}$ in hydroponics. EL was significantly lower in the $\mathrm{H}-2$ plants. Each bar represents the mean \pm SE $(n=6)$. Different letters in each graph $(a-d)$ indicate significant differences $(\mathrm{p}<0.05$, ANOVA and DMRT). 
for the production of new cultivars containing abiotic stress tolerant genes as well as economically important traits, continues today (Hajjar et al. 2007). In this paper we have presented two hybrids that have intermediate characteristics between both parents and will likely be useful in rice breeding. The most remarkable morphological changes observed in the Latisail $(4 \mathrm{x}) \times P$. coarctata hybrid plants presented here were their intermediate heights and seed morphology suggesting the pleiotropic effect of hybridization (Fig. 2). In fact, the entire plant phenotype was changed although the species-specific characteristics of the cultivated parent Latisail (2x) prevailed. We also observed modified traits related to salt tolerance in hybrid plants as they were altered by the probable intergeneric genome transfer. In physiological analysis at seedling stage under salinity (150 mM salt), LDS (leaf damage score), root length, chlorophyll content

Table 1. Per cent seed set and survival in crossing of $O$. sativa and P. coarctata.

\begin{tabular}{lllcc}
\hline $\begin{array}{l}\text { Sl. } \\
\text { No. }\end{array}$ & $\begin{array}{c}\text { Cross } \\
\text { combination }\end{array}$ & $\begin{array}{l}\text { \% seed } \\
\text { set }\end{array}$ & $\begin{array}{c}\text { Germinated plant } \\
\text { (No.) }\end{array}$ & $\begin{array}{c}\text { Surviving plant } \\
\text { (No.) }\end{array}$ \\
\hline 1 & Lat $(4 \mathrm{x}) /$ P. coarctata & $2.31(865)$ & 12 & 2 \\
2 & BR7 $(4 \mathrm{x}) /$ P. coarctata & $0.0(157)$ & - & - \\
3 & P. coarctata/BR7 $(4 \mathrm{x})$ & $0.0(36)$ & - & - \\
4 & P. coarctata/BR43 (2x) & $0.0(13)$ & - & - \\
5 & IR64 $(4 \mathrm{x}) /$ P. coarctata & $0.0(77)$ & - & - \\
6 & P. coarctata/ IR64 (4x) & $0.0(12)$ & - & - \\
7 & IR36 $(4 \mathrm{x}) /$ P. coarctata & $0.0(31)$ & - & - \\
\hline
\end{tabular}

Values in parentheses indicate total number of florets used in crossing.

and electrolyte leakage were measured. In all four experiment putative hybrids showed significantly better results than maternal parent Latisail $(4 \mathrm{x})$ but putative hybrid $\mathrm{H}-2$ showed significantly better result than other putative hybrid $(\mathrm{H}-1)$ (Figs 4 and 5). Furthermore, in rice specific SSR markers based PCR both of the hybrid showed heterozygote banding pattern in three SSR markers (RM-581, RM-20224 and RM- 25271) out of 36 RM markers tested (Fig. 3). From the bulbosum technique it had been known that during interspecific hybridization some of the genes/portion of genes from one species are transferred to another species and remained by chromatin introgression but due to genetic incompatibility uniparental chromosome might be lost (Gernand et al. 2005). So from the banding pattern of hybrids for RM markers we can hypothesize that some of the genes of $P$. coarctata were introduced into hybrids and some recombination occurred before its incompatible chromosome might have become lost. So from this SSR marker analysis and physiological screening it could be 
said that some of the salt tolerant alleles of $P$. coarctata introgressed into Latisail (4x) because these types of banding pattern and physiological characteristics under salt stress are only possible if successful hybridization had initially occurred.

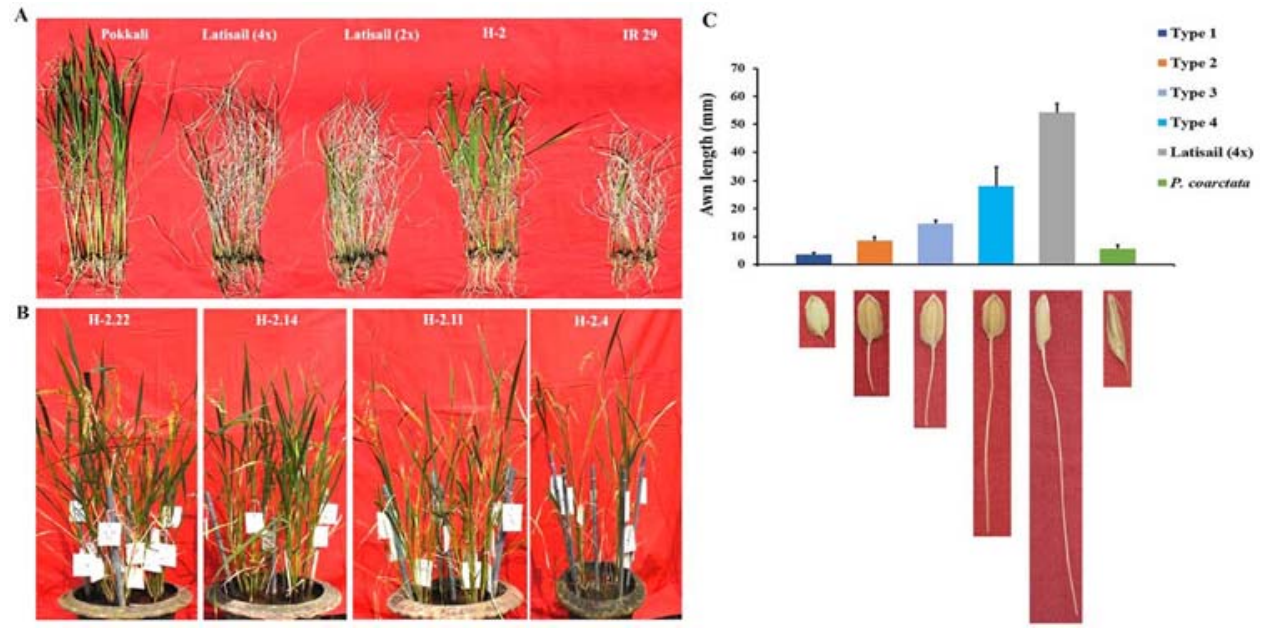

Fig. 6. Survival test of $\mathrm{H}-2$ hybridsat $\mathrm{F}_{2}$ generation. A. Phenology of the hybrids and Latisail plants after $15 \mathrm{dS} / \mathrm{m} \mathrm{NaCl}$ stress in hydroponics (From left to right: Pokkali, Latisail (4x), Latisail (2x), H-2 and IR29). B. Phenology of the H-2 hybrids at $F_{2}$ (after planting in soil) which survived after application of $15 \mathrm{dS} / \mathrm{m} \mathrm{NaCl}$ stress in hydroponics. Each plant was different from each other regarding their height and seed appearance. C. Awn length of $\mathrm{H}-2$ hybrids was significantly different from Latisail $(4 x)$. Each bar represents the mean $\pm S E(n=6)$.

The results suggest that in the future massive hybridization programme using Latisail $(4 \mathrm{x})$ as female can be undertaken and maybe the hybrids produced repeatedly crossed with $P$. coarctata with the hope of obtaining hybrid seeds with novel recombinants from the halophyte genome. More crosses between $P$. coarctata and other local cultivars grown in the moderately saline coastal belt will also be attempted in the light of finding more salt tolerant new cultivars and attempts made to advance generations in order to stabilize the genomes for attaining homozygosity.

\section{Acknowledgement}

Funds for this study were received from the Bangladesh Climate Change Trust (BCCT), Ministry of Environment and Forests, Bangladesh which is most gratefully acknowledged. 


\section{References}

Amin M, Elias SM, Hossain A, Ferdousi A, Rahman MS, Tuteja N and Seraj ZI (2012) Over-expression of a DEAD-box helicase, PDH45, confers both seedling and reproductive stage salinity tlerance to rice (Oryza sativa L.). Molecular Breeding 30: 345-354.

Blakeslee AF and Avery AG (1937) Methods of inducing doubling of chromosomes in plants by treatment with colchicine. Journal of Heredity. 28: 393-411.

Cox T, Bender M, Picone C,Tassel DV, Holland J, Brummer E, Zoeller B, Paterson A and Jackson W (2002) Breeding perennial grain crops. Critical Reviews in Plant Sciences. 21: 59-91.

Doyle JJ (1987) A rapid DNA isolation procedure for small quantities of fresh leaf tissue. Phytochem bull. 19: 11-15.

Fedak G (1985) Alien species as sources of physiological traits for wheat improvement. Euphytica. 34: 673-680.

Flowers TJ, Flowers SA, Hajibagheri MA and Yeo AR (1990) Salt tolerance in the halophytic wild ricePorteresia coarctata Tateoka. New Phytol. 114: 675-684.

Garg R, Verma M, Agrawal S, ShankarR, Majee M and Jain M (2013) Deep Transcriptome Sequencing of Wild Halophyte Rice, Porteresiacoarctata, Provides Novel Insights into the Salinity and Submergence Tolerance Factors. DNA Research. $1-16$.

Gernand D, RuttenT, VarshneyA, Rubtsova M, Prodanovic S, Brüß C, Kumlehn J, Matzk F and Houben A (2005) Uniparental chromosome elimination at mitosis and interphase in wheat and pearl millet crosses involves micronucleus formation, progressive heterochromatinization, and DNA fragmentation. The Plant Cell. 17: 2431-2438.

Goodman RM, Hauptli H, Crossway A and Knauf VC (1987) Gene transfer in crop improvement. Science. 236: 48-54.

Hajjar R and Hodgkin T(2007) The use of wild relatives in crop improvement: a survey of developments over the last 20 years. Euphytica. 156: 1-13.

Jelodar N, Blackhall N, Hartman T, Brar D, Khush G, Davey M, Cocking E and Power J (1999) Intergeneric somatic hybrids of rice [Oryza sativa L.(+) Porteresia coarctata (Roxb.) Tateoka]. Theoretical and applied genetics. 99: 570-577.

Latha R, Rao CS, Subramaniam H, Eganathan P and Swaminathan M (2004) Approaches to breeding for salinity tolerance-a case study on Porteresia coarctata. Annals of applied biology. 144: 177-184.

Liu K and Muse SV (2005) PowerMarker: an integrated analysis environment for genetic marker analysis. Bioinformatics. 21: 2128-2129.

Menguer PK, Sperotto RA and Ricachenevsky FK (2017) A walk on the wild side: Oryza species as source for rice abiotic stress tolerance. Genetics and Molecular Biology.Genetic and Molecular Biology. 40:238-252

Rick CM, De Verna JW, Chetelat RT and Stevens MA(1986) Meiosis in sesquidiploid hybrids of Lycopersicon esculentum and Solanum lycopersicoides. Proceedings of the National Academy of Sciences. 83: 3580-3583. 
Sarker RH, Samad MA, Seraj ZI, Hoque MI and Islam AS (1993) Pollen tube growth in crosses between Porteresia coarctata and Oryza sativa. Euphytica 69: 129-134.

Sengupta S and Majumder AL (2009) Insight into the salt tolerance factors of a wild halophytic rice, Porteresia coarctata: A physiological and proteomic approach. Planta. 229: 911-929.

Sengupta S and Majumder AL (2010) Porteresia coarctata (Roxb.) Tateoka, a wild rice: a potential model for studying salt-stress biology in rice. Plant Cell and Environment. 33: 526-542.

Seraj ZI, Faruque MO, Hossain KG, Sarker RH, Devi T and Islam AS (1996) Attempted hybridizaion between Oryza sativa L. and P. coarctata T. Inter Rice Res. Notes 21: 35.

Sneath PHA and Sokal RR (1973) Numerical Taxonomy. The principle and practice of numerical classification, Freeman, San Francisco, Calif, USA, 1973.

Vassilevska-Ivanova R, Kraptchev B, Stancheva I, Geneva M, Iliev I and Georgiev G (2014) Utilization of related wild species (Echinacea purpurea) for genetic enhancement of cultivated sunflower (Helianthus annuus L.). Turkish Journal of Agriculture and Forestry 38: 15-22.

Yasmin F, Biswas S, Jewel GNA, Elias SM and Seraj ZI (2016) Constitutive Overexpression of the Plasma Membrane $\mathrm{Na}^{+} / \mathrm{H}^{+}$Antiporter for Conferring Salinity Tolerance in Rice. Plant Tissue Culture and Biotechnoy 25: 257-272.

Yoshida S, Forno D, Cock J and Gomez K (1976) Routine procedure for growing rice plants in culture solution. Laboratory manual for physiological studies of rice., International Rice Resararch Institute, Phillipines 61-66. 